\title{
LETTERS
}

\section{Dyspnoea and a lung mass in a young female 2 weeks after Caesarean delivery}

\section{To the Editors:}

A 28-yr-old female presented 2 weeks after an uneventful delivery by Caesarean section with progressive cough, dyspnoea and left-sided chest pain. Medical history was uneventful but she had smoked until her pregnancy (16 pack-yrs). On admission, conventional chest radiography revealed a large pulmonary mass in the left lung (fig. 1a). A computed tomography (CT) scan showed a large, slightly inhomogeneous mass in the upper lobe of the left lung (fig. 1b). Apart from a 17-mm lesion in the left pulmonary vein, no other metastases were found. CT-guided true-cut biopsy was not diagnostic, and so a thoracotomy was performed. In the frozen section, a malignant process was diagnosed, and the surgeon proceeded to a left upper lobectomy and mediastinal lymphadenectomy.

The histological workup of the mostly necrotic and partly cystic well-demarcated mass showed a biphasic pattern composed of branching glandular structures and cords resembling fetal lung, set in an undifferentiated stroma with polygonal and spindled cells (fig. 1c-e). The mesenchymal cells tended to be more closely packed around the epithelial tubules. High proliferation activity (Ki67 up to 90\%) in both elements of this true mixed tumour underlined the malignant quality of the epithelial component and sarcomatous stroma. The patient was thus diagnosed with a biphasic pulmonary blastoma.

2 days after surgery, the patient developed a left-sided haemiparesis. A brain CT showed multiple ischaemic lesions but no evidence of metastases. The origin of the emboli was suspected to be persistent tumour growth along the left pulmonary vein reaching the left atrium. The patient was transferred to a cardiosurgical centre where the remaining tumour was resected successfully. The neurological deficits resolved spontaneously and completely.

Post-operative restaging including a CT scan of the brain (the patient refused to undergo brain magnetic resonance imaging (MRI)) showed no evidence of persistent tumour manifestations. In view of the clinical diagnosis of tumour emboli and the young age of the patient, we decided to administer an adjuvant chemotherapy for 6 months. The IVADo-regimen (ifosfamide, vincristin, actinomycin D and doxorubicin), which is used in children with pleuropulmonary blastoma [1], was chosen initially. After 3 months the regimen was changed to cisplatin/ifosfamide in view of the epithelial tumour component. At the end of the chemotherapy, further workup including brain CT showed no evidence of disease. At a scheduled follow-up visit of the asymptomatic patient 3 months later, a solitary brain metastasis was detected on the right parietal side (brain CT and MRI). Because surgical resection was suspected to have a high risk of permanent neurological deficits, the patient received stereotactic radiosurgery to the metastasis and, because of the history of multiple tumour emboli, whole-brain radiotherapy (30 Gy).
18 months later, the patient remains in very good general condition and without evidence of disease recurrence in repeated CT and MRI examinations.

Biphasic pulmonary blastoma is a rare malignancy, representing $<1 \%$ of all lung cancers [2]. The first description of pulmonary blastoma was published in 1945 by BARRETT and BARNARD [3], when the disease was initially named pulmonary embryoma because of its microscopic resemblance to embryonic lung. Pulmonary blastoma is nowadays subdivided into three categories: classic biphasic pulmonary blastoma; welldifferentiated fetal adenocarcinoma; and pleuropulmonary blastoma [4]. The latter is a malignant stromal tumour of early childhood and rarely occurs after the age of 5 yrs.

Biphasic pulmonary blastoma is composed of malignant primitive embryonal glands and mesenchyme. Well-differentiated fetal adenocarcinoma shows malignant glands that resemble fetal lung at the 10th to 16th week of gestation. The mesenchyme is histologically benign. Diagnosis and differentiation are often difficult, and expert opinion should be sought. Our case was assessed by L. Dehner (Washington University, St Louis, MO, USA), one of the most experienced pathologists in this disease entity [5]. He confirmed the diagnosis.

Pulmonary blastomas can occur at any age, with a median of 35-40 yrs at diagnosis [6]. Patients present with nonspecific respiratory symptoms (cough, haemoptysis, dyspnoea, chest pain) that may mimic a respiratory tract infection. In $40 \%$ of cases, the tumours are found by routine chest radiographs in asymptomatic patients. Tumour size and location are very variable and without predominance. Primary tumours with $<5 \mathrm{~cm}$ maximum dimension have a more favourable prognosis [6]. In the only case series with documented smoking habits, up to $80 \%$ of patients had a smoking history [6]. The prognosis of pulmonary blastoma in general is poor. A historical case review revealed that patients with biphasic pulmonary blastomas have poorer prognosis than those with monophasic neoplasms; $52 \%$ of patients with biphasic blastoma died of their disease, but only $14 \%$ of patients suffering from well-differentiated fetal adenocarcinoma [6]. This finding has been confirmed in a smaller case series [7].

The diagnosis of this tumour shortly after pregnancy raises the question of whether female sex hormones might play a role in the development of the disease. Well-differentiated fetal adenocarcinomas express the cell-adhesion molecule $\beta$-catenin, which forms a complex with steroid hormone receptors. A study therefore hypothesised that oestrogens might play a role in the development of the disease [8]. In a single case report, a well-differentiated fetal adenocarcinoma was found 3 months after delivery [9]. However, in view of the reported equal sex distributions for these tumours and the fact that it is a very rare 

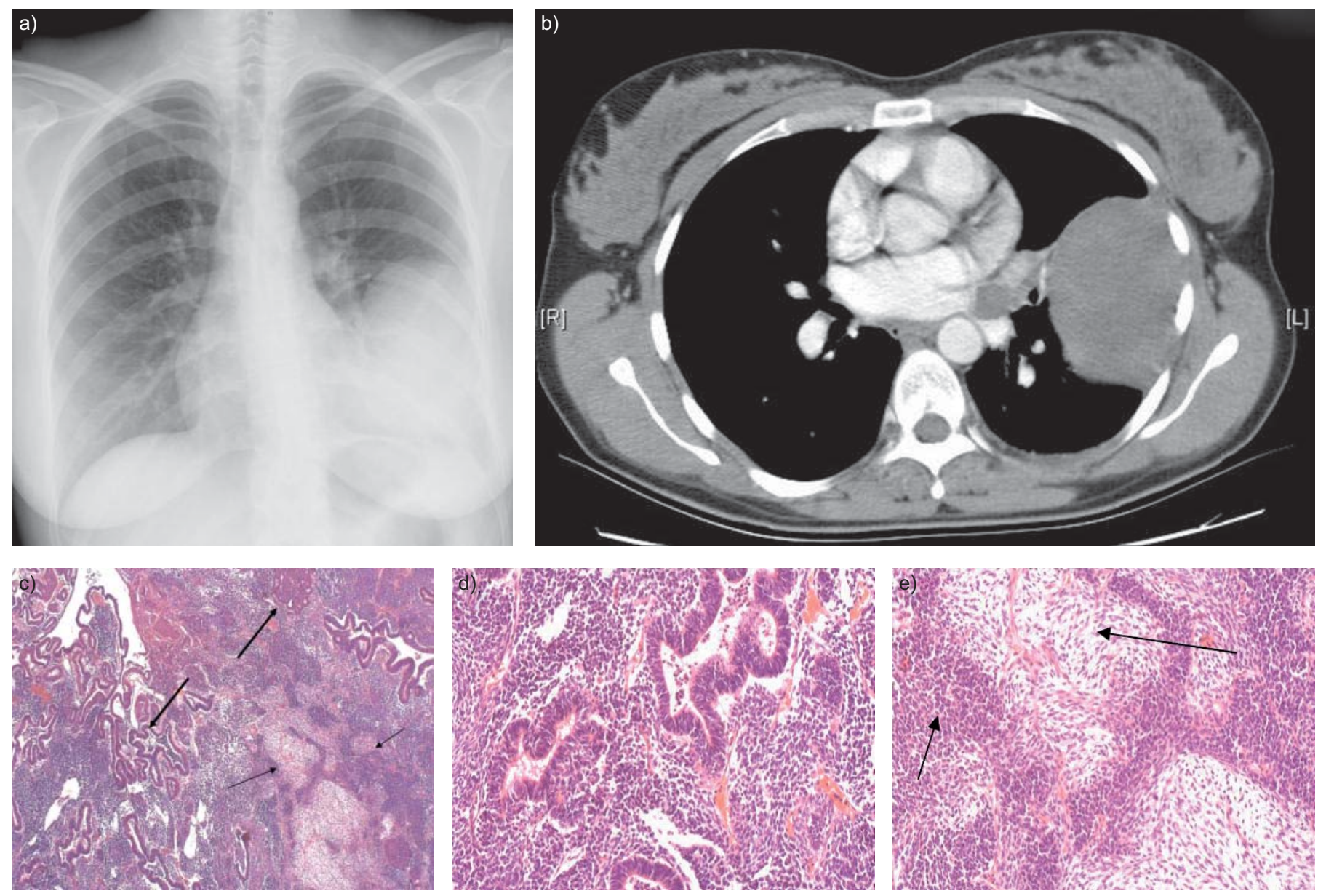

FIGURE 1. a) Chest radiography and b) computed tomography images of the patient on admission. c-e) Haemotoxylin and eosin stained sections of the biphasic neoplasm composed of c) epithelial glandular tubular structures (thick arrows) and a sarcomatous mesenchymal stroma (thin arrows) (50 $\times$ magnification), d) the epithelial elements resemble fetal lung $(100 \times$ magnification) and e) the undifferentiated stroma is formed of loosely arranged polygonal cells (short arrow) and more spindle-shaped cells forming bands and nodules (long arrow) $(100 \times$ magnification)

disease, a relationship between pulmonary blastoma and pregnancy appears very difficult to support.

The primary treatment of choice for localised disease is surgical resection and in case of complete resection, cure can be achieved. The role of adjuvant chemotherapy or radiotherapy is controversial due to a lack of data. Based on a review of the literature, CUTLER et al. [10] recommended radiotherapy, and chemotherapy with cisplatin and etoposide as an effective regimen after surgery.

Brain metastases are very rare in this disease. The first report of brain metastasis of pulmonary blastoma was reported by BARSON et al. [11] in 1968. A review of the literature on this topic found six cases of pulmonary blastoma with brain metastasis [12]. Patients who underwent no further treatment survived for 2.5 and 4 months. Patients with brain metastases who received treatment (surgery, radiotherapy or chemotherapy) survived for 9-25 months.

Extension of pulmonary blastoma into the left atrium, another feature of this case, is also exceedingly rare and has only been published once before [13].
In conclusion, the optimal treatment of patients with pulmonary blastoma remains unclear but initial complete resection represents the most important aspect, and can be curative in localised disease. Treatment of metastatic disease should consider the possibility of further resection in case of solitary lesions. Our case demonstrates that long-term survival is possible in this rare disease with generally poor prognosis if an aggressive approach is undertaken. However, it is crucial to gain further knowledge to improve therapy for this rare malignancy.

\section{Schwitter*, P. Potocnik", R. von Moos*, H. Frick", M. Furrer ${ }^{*}$ and R. Cathomas*}

Depts of *Medical Oncology "Surgery and "Pathology, Kantonsspital Graubünden, Chur, Switzerland.

Correspondence: R. Cathomas, Dept of Medical Oncology, Kantonsspital Graubünden, CH-7000 Chur, Switzerland. E-mail: richard.cathomas@ksgr.ch

Statement of Interest: None declared. 


\section{REFERENCES}

1 Bisogno G, Ferrari A, Bergeron C, et al. The IVADo regimen - a pilot study with ifosfamide, vincristine, actinomycin D, and doxorubicin in children with metastatic soft tissue sarcoma. Cancer 2005; 103: 1719-1724.

2 Kliem V, Bugge M, Leimenstoll K, et al. Pulmonary blastoma - a rare tumour. Clin Investig 1992; 70: 927-931.

3 Barret NR, Barnard WG. Some unusual thoracic tumours. Br J Surg 1945; 32: 447-457.

4 Koss M, Travis W, Moran C. Pulmonary sarcomas, blastomas, carcinosarcomas and teratomas. In: Hasleton PS, ed. Spencer's Histopathology of the Lung. 5th Edn. New York, McGraw Hill, 1996; pp. 1092-1100.

5 Priest JR, Hill DA, Williams GM, et al. Type I pleuropulmonary blastoma; a report from the International Pleuropulmonary Blastoma Registry. J Clin Oncol 2006; 24: 4492-4498.

6 Koss MN, Hochholzer L, O'Leary T. Pulmonary blastomas. Cancer 1991; 67: 2368-2381.

7 Zaidi A, Zamvar V, Macbeth F, et al. Pulmonary blastoma; medium-term results from a regional center. Ann Thorac Surg 2002; 73: 1572-1575.
8 Nakatani Y, Masudo K, Nozawa A, et al. Biotin-rich optically clear nuclei express estrogen receptor beta; tumors with morules may develop under the influence of estrogen and aberrant beta-catenin expression. Human Pathol 2004; 35: 869-874.

9 Esper A, Force S, Gal A, et al. A 36-year old woman with hemoptysis and a lung mass 3 months after delivery. Chest 2006; 130: 1620-1623.

10 Cutler CS, Michel RP, Yassa M, et al. Pulmonary blastoma; case report of a patient with a 7-year remission and review of chemotherapy experience in the world literature. Cancer 1998; 82: 462-467.

11 Barson AJ, Jones AW, Lodge KV. Pulmonary blastoma. J Clin Pathol 1968; 21: 480-485.

12 Kouvaris JR, Gogou PV, Papacharalampous XN, et al. Solitary brain metastasis from classic biphasic pulmonary blastoma; a case report and review of the literature. Onkologie 2006; 29: 568-570.

13 Zavala-Alarcon E, Sudhakar S, Gonzales LR, et al. Extension of pulmonary blastoma into the left atrium. Mayo Clin Proc 2001; 76: 657-660.

DOI: $10.1183 / 09031936.00187210$

\section{Tuberculosis in the elderly in Germany}

\section{To the Editors:}

Germany has a low tuberculosis (TB) incidence with a decreasing trend [1].

However, the proportion of TB patients aged $\geqslant 60 \mathrm{yrs}$ is increasing (1976: 26.5\%; 1986: 30.9\%; 1996: 33.7\%; 2006: 34.8\%. 1976 and 1986 figures are for the Federal Republic of Germany only), reflecting demographic changes. Age-related factors not only increase the risk of $\mathrm{TB}$ reactivation but also enhance susceptibility to TB infection, abetting outbreaks, e.g. in nursing homes $[2,3]$. To optimise healthcare services and TB control in this subpopulation of growing impact, detailed knowledge of the epidemiological features of TB in the elderly is needed.

National German TB notification data from 2002 to 2006 were analysed as a pooled 5-yr data set stratified into two age groups: TB patients 15-59 yrs (referred to as "younger adults") and those aged $\geqslant 60$ yrs ("the elderly"). For both groups, proportions of TB cases with reference to demographic factors, affected organs, drug resistance, case finding and treatment outcome were determined. Mortality (based on treatment outcome notification data), TB incidence, and age-specific TB incidence in 5-yr strata were calculated based on population data from regional statistical offices (time averaged 2002-2006). As the country of birth was unknown for the general population, origin-related TB incidence was derived from citizenship information. Proportion (\%) and incidence (cases per 100,000 population) were compared between both groups using the Z-test. Odds ratios (ORs), 95\% confidence intervals (CIs) and corresponding p-values were specified. Data were collected using the electronic reporting system SurvNet@rki (Robert Koch Institute, Berlin, Germany) and analysed using SPSS version 15 (SPSS Inc., Chicago, IL, USA), Excel (Microsoft Corporation, Redwood, CA, USA) and Stata version 11.0 (StataCorp LP, College Station, TX, USA).
A total of 31,459 TB cases aged $\geqslant 15$ yrs were identified. Details of the study set are presented in table 1 .

The proportion of elderly patients $(36.0 \%)$ remained stable over the study period. The elderly differed from the younger TB cases, first of all in patient origin: $45.0 \%$ of the younger TB cases had a foreign citizenship and $56.6 \%$ were born abroad, versus $12.0 \%$ and $23.1 \%$, respectively, of the elderly patients.

Proportions of pulmonary TB (PTB), which was the most common form, and of extrapulmonary TB (EPTB) were almost similar in the two age groups. However, the EPTB forms differed: genitourinary TB was more often diagnosed among the elderly, especially in males, while extrathoracic lymph node TB was seen less frequently. Among PTB patients, both culturepositivity rate and positive microscopy results were seen significantly less frequently in the elderly (76.2 versus $79.1 \%$, OR 0.84 (95\% CI 0.79-0.90), $\mathrm{p}<0.001$ and $38.5 \%$ versus $44.7 \%$, OR 0.78 (95\% CI 0.73-0.83), $\mathrm{p}<0.001$, respectively).

Drug resistance rates were markedly lower in the elderly for any drug resistance (6.5 versus $13.9 \%$, OR 0.43 (95\% CI $0.39-0.48)$, $\mathrm{p}<0.001)$ and for multidrug resistance $(0.6$ versus $3.1 \%$, OR 0.18 $(95 \%$ CI $0.13-0.25), p<0.001)$. Drug resistance rates in the foreignborn elderly exceeded those among German-born patients by a factor of 2-3, although the discrepancy was less pronounced than in younger adults. However, the pyrazinamide resistance rate was higher in the elderly (3.2 versus $2.6 \%$, OR $1.28(95 \% \mathrm{CI}$ $1.07-1.52), p=0.006)$ and more frequent in the German-born elderly than in the foreign-born elderly $(3.8 \%(n=188)$ versus $1.7 \%(n=27))$.

Fewer elderly patients than younger patients were diagnosed via active case finding (5.9 versus $18.4 \%$, OR 0.28 (95\% CI $0.25-$ $0.31), \mathrm{p}<0.001)$, including contact tracing (1.6 versus $6.9 \%$, OR $0.21(95 \%$ CI $0.18-0.26))$. TB as a post mortem diagnosis was 